\section{VALIDATION OF THE SUPERPARAMAGNETIC IRON OXIDE TRACER FOR THE DETECTION OF THE SENTINEL NODE IN VULVAR CANCER}

S Huerta Martín*, C Blasco de Imaz Alonso, D Del Valle, A Lekuona Artola, R Ruiz, P Cobas, AP Gurría De la Torre, M Gorostidi, I Jaunarena, J Céspedes Manterola. Donostia's University Hospital, San Sebastián, Spain

\subsection{6/ijgc-2021-ESGO.631}

Introduction/Background* Surgical lymph node staging is required in vulvar cancer in patients candidates for primary surgery. In order to complete the diagnosis and reduce the morbidity due to radical inguinal lymphadenectomy, the selective sentinel lymph node biopsy (SLNB) has been validated reporting a false negative rate of $2-6 \%$. This technique avoids high morbidity and achieves diagnostic precision. SLNB should be the standard treatment when indicated, since it is a safe technique with less morbidity.

The tracer validated in SLNB is technetium-99m (Tc-99). In Donostia's University Hospital (DUH), we are carrying out a study to validate the Superparamagnetic iron oxide tracer (SPIONs) in vulvar cancer compared to the currently standarized.

Methodology This is a prospective observational study at the DUH from April 2016 to the present day using a sample size of 12 patients.

Information was obtained from DUH's database and analysed with SPSS.

Eligibility criteria to perform SLNB were squamous histology, FIGO Ib-II stage, size $\leq 4$ centimeters, unifocal injury negative and clinical-radiological lymph node examination, all defined in GROINSS-V.

Subjects received both tracers, the one already validated (Tc-99) and the tracer under study (SPIONs). The sentil nodes detected were analyzed intraoperatively. If the result of the intraoperative analysis was positive or the sentil node was not detected, a radical inguinal homolateral lymphadenectomy was performed.

Result(s)* Twelve patients were included. Sentinel node detection rate was a $100 \%$.

Two patients (16.67\%) (after performing SLNS) had a positive result. The nodes with a histopathological positive result were always been elected as sentinel node. In patients with positive nodes, the rest of the accessory lymph nodes obtained from the lymphadenectomy were negative being therefore the percentage of false negatives 0 .

Conclusion* SPIONs is showing not to be inferior to Technetium-99m for SLNB, with 13 sentil nodes left to reach the sample size recommended in order to complete the study.

Comparing to Technetium-99m, SPIONs has the following benefits: injection during the surgery procedure, not need to go to a nuclear medicine room previously, avoid pain caused by the injection of Technetium-99m and finally it can be used in hospitals without nuclear medicine service.

\section{THE VULVAR IMMUNOHISTOCHEMICAL PANEL (VIP) PROJECT: MOLECULAR PROFILES OF VULVAR SQUAMOUS CELL CARCINOMA}

$1,2^{2} \mathrm{G}$ Garganese, ${ }^{3} \mathrm{FS}$ Inzani, ${ }^{4} \mathrm{G}$ Mantovani, ${ }^{5} \mathrm{SM}$ Fragomeni, ${ }^{6} \mathrm{~L}$ Della Corte, ${ }^{3} \mathrm{~A}$ Piermattei, ${ }^{3} \mathrm{~A}$ Santoro, ${ }^{3} \mathrm{G}$ Angelico, ${ }^{7} \mathrm{~L}$ Giacò, ${ }^{5} \mathrm{G}$ Corrado, ${ }^{2} \mathrm{~S}$ Bove, ${ }^{2} \mathrm{~A}$ Romito, ${ }^{1,5} \mathrm{~A}$ Fagotti, ${ }^{3} \mathrm{GF}$ Zannoni, $1,5 \mathrm{G}$ Scambia. 'Università Cattolica del Sacro Cuore, Istituto di Clinica Ostetrica e Ginecologica, Rome, Italy, ${ }^{2}$ Mater Olbia Hospital, Gynecology and Breast Care Center, Olbia, Italy; ${ }^{3}$ Fondazione Policlinico Universitario A. Gemelli IRCCS, Unità di GinecoPatologia e Patologia Mammaria, Dipartimento Scienze della Salute della Donna, del Bambino e di Sanità Pubblica, Rome, Italy; ${ }^{4}$ RCCS Ospedale Sacro Cuore Don Calabria, Chirurgia Pelvica Mini-Invasiva, International School of Surgical Anatomy, Negrar di Valpolicella, Italy; ${ }^{5}$ Fondazione Policlinico Universitario A. Gemelli IRCCS, Unità di Ginecologia Oncologica, Dipartimento Scienze della Salute della Donna, del Bambino e di Sanità Pubblica, Rome, Italy; ${ }^{6}$ University of Naples Federico II, Department of Neuroscience, Reproductive Sciences and Dentistry, School of Medicine, Naples, Italy; ${ }^{7}$ Fondazione policlinico Universitario A. Gemelli IRCCS, Rome, Italy

\subsection{6/ijgc-2021-ESG0.632}

Introduction/Background* Investigate the immunohistochemical (IHC) expression of biological markers as potential prognostic/therapeutic factors in vulvar squamous cell carcinoma (VSCC).

Methodology A series of 101 patients surgically treated at our Center from 2016 to 2020 were retrospectively enrolled: 53 node negative (Group A) and 48 node positive (Group B). A total of 146 samples, 101 primary tumor (T) and 45 nodal metastasis $(\mathrm{N})$, were investigated. The IHC

\begin{tabular}{|c|c|c|c|c|c|c|c|c|c|c|c|}
\hline \multirow[t]{2}{*}{$\mathbf{N}^{\circ}$} & \multirow[t]{2}{*}{ Age } & \multirow[t]{2}{*}{ G } & \multirow[t]{2}{*}{ FIGO } & \multirow{2}{*}{$\begin{array}{l}\text { Size } \\
(\mathrm{cm})\end{array}$} & \multirow[t]{2}{*}{ Technique } & \multirow[t]{2}{*}{ Laterality } & \multicolumn{2}{|c|}{ Right side } & \multicolumn{2}{|c|}{ Left side } & \multirow[t]{2}{*}{ Lymphadenectomy } \\
\hline & & & & & & & SLNB & $\mathrm{PA}^{*}$ & SLNB & $\mathrm{PA}^{*}$ & \\
\hline 1 & 62 & 1 & ॥ & 2.3 & Vulvectomy & Unilateral & & & 2 & Negative & \\
\hline 2 & 80 & 2 & IB & 2.6 & Vulvectomy & Bilateral & 1 & Negative & 2 & Negative & \\
\hline 3 & 70 & 2 & IB & 1.5 & Hemivulvectomy & Bilateral & 2 & Positive & 1 & Positive & $\begin{array}{l}\text { Bilateral } \\
29 \text { Nodes }\end{array}$ \\
\hline 4 & 68 & 1 & IB & 3 & Vulvectomy & Bilateral & 2 & Negative & 1 & Negative & \\
\hline 5 & 45 & 2 & IB & 2.1 & Hemivulvectomy & Bilateral & 2 & Negative & 1 & Negative & \\
\hline 6 & 55 & 2 & IB & 2 & Hemivulvectomy & Bilateral & 1 & Negative & 1 & Positive & $\begin{array}{l}\text { Unilateral } \\
5 \text { Nodes }\end{array}$ \\
\hline 7 & 53 & 1 & IB & 0.5 & Wide local excision & Unilateral & & & 2 & Negative & \\
\hline 8 & 78 & 2 & IB & 1.4 & Vulvectomy & Bilateral & 2 & Negative & 1 & Negative & \\
\hline 9 & 78 & 1 & IB & 1 & Wide local excision & Unilateral & 1 & Negative & & & \\
\hline 10 & 53 & 2 & IB & 3 & Vulvectomy & Bilateral & 1 & Negative & 1 & Negative & \\
\hline 11 & 79 & 1 & IB & 2 & Vulvectomy & Bilateral & 1 & Negative & 1 & Negative & \\
\hline 12 & 64 & 2 & IB & 3 & Vulvectomy & Unilateral & 1 & Negative & & & \\
\hline
\end{tabular}

*Pathological Anatomy 
panel included: p16, p53, MLH1, MSH2, MSH6, PMS2, PD-L1, CD3, HER2/neu, ER, PR, EGFR, VEGF and CD31. The reactions were evaluated on qualitative and semi-quantitative scale. Generalized Linear Model (GLM) and Cluster analysis were performed in $\mathrm{R}$ statistical environment. A distance plot compared the IHC panel of $\mathrm{T}$ with the correspondent $\mathrm{N}$.

Result(s)* Mismatch repair proteins (MMR), ER, PR and HER2/neu were excluded from data analysis because of homogeneous expression in all samples. Group A: the p16positive expression (surrogate of HPV-dependent pathway) was significantly higher $(20.8 \%$ vs $6.2 \%, p=0.04)$. Group B: PD-L1-positive and high EGFR expression were found respectively in $77.1 \%$ and $97.9 \%$ patients, ( $T$ and/or $\mathrm{N}$ ). Overall, p16-negative tumors showed a higher PD-L1 expression $(60.9 \%$ vs. $50.0 \%)$. In both groups tumoral immune infiltration (CD3 expression), was mainly moderate/intense $(80 \%$ vs. 95\%). VEGF showed strong/moderate-diffuse expression in $13.9 \%$ of $\mathrm{T}$ samples. CD31 was used to study tumoral micro vessel density (MVD) with no difference between Group A and Group B. p53 and PD-L1 showed a significant association with nodal metastasis. Odds ratio (OR) for p53 mutation was 4.26 (CI 95\% $=1.14-15.87, \mathrm{p}=$ 0.03 ); OR for PD-L1 positivity was 2.68 (CI 95\% $=1.0-$ 7.19, $\mathrm{p}<0.05)$.

The cluster analysis identified 3 and 4 sub-groups of molecular profiles respectively in Group A and B, with no different prognosis. Moreover, the molecular profile of each $\mathrm{N}$ and corresponding $\mathrm{T}$ diverged significantly in $18 / 41$ (43.9\%) cases.

Conclusion* These results support a potential role of immune checkpoint inhibitors and anti-EGFR drugs in a subset of patients with VSCC, especially with worse prognosis (metastatic, HPV-independent). It is mandatory to repeat the panel in the metastatic site to identify changes of marker expression.

\section{UPDATE OF THE GROSNAPET STUDY: ON THE WAY TO OVERCOME SENTINEL NODE LIMITS IN VULVAR CANCER}

\begin{abstract}
${ }^{1} \mathrm{SM}$ Fragomeni* ${ }^{*}{ }^{2} \mathrm{~A}$ Collarino, $2,3 \mathrm{~V}$ Rufini, ${ }^{1} \mathrm{~A}$ Federico, ${ }^{4} \mathrm{~F}$ Inzani, ${ }^{1} \mathrm{G}$ Corrado, ${ }^{5,6} \mathrm{~S}$ Gentileschi, ${ }^{7} \mathrm{~L}$ Tagliaferri, ${ }^{8} \mathrm{D}$ Verri, ${ }^{9} \mathrm{~A}$ Zampolini Faustini, ${ }^{2} \mathrm{~A}$ Florit, ${ }^{1,9} \mathrm{~A}$ Fagotti, ${ }^{1,9} \mathrm{G} \mathrm{Scambia},{ }^{8,9} \mathrm{G}$ Garganese. ${ }^{1}$ Fondazione Policlinico Universitario A. Gemelli IRCCS, Unità di Ginecologia Oncologica, Dipartimento Scienze della Salute della Donna, del Bambino e di Sanità Pubblica, Rome, Italy; ${ }^{2}$ Fondazione Policlinico Universitario A. Gemelli IRCCS, Unità di Medicina Nucleare, Dipartimento di diagnostica per Immagini, Radioterapia Oncologica ed Ematologia, Rome, Italy; ${ }^{3}$ Università Cattolica del Sacro Cuore, Unità di Medicina Nucleare, Dipartimento di diagnostica per Immagini, Radioterapia Oncologica ed Ematologia, Rome, Italy; ${ }^{4}$ Fondazione Policlinico universitario Agostino Gemelli IRCCS, Unità di GinecoPatologia e Patologia Mammaria, Dipartimento Scienze della Salute della Donna, del Bambino e di Sanità Pubblica, Rome, Italy; ${ }^{5}$ Università Cattolica del Sacro Cuore, Istituto di Clinica Chirurgica, Rome, Italy; ${ }^{6}$ Fondazione Policlinico Universitario A. Gemelli IRCCS, Unità di Chirurgia Plastica, Dipartimento Scienze della Salute della Donna, del Bambino e di Sanità Pubblica, Rome, Italy; ${ }^{7}$ Fondazione Policlinico Universitario A. Gemelli, IRCCS, Unità Operativa Complessa di Radioterapia, Dipartimento di Scienze Radiologiche, Radioterapiche ed Ematologiche, Rome, Italy; ${ }^{8}$ Mater Olbia Hospital, Gynecology and Breast Care Center, Olbia, Italy: ${ }^{9}$ Università Cattolica del Sacro Cuore, Istituto di Clinica Ostetrica e Ginecologica, Rome, Italy
\end{abstract}

\subsection{6/ijgc-2021-ESG0.633}

Introduction/Background* Sentinel node biopsy (SNB) is the standard of care for vulvar cancer patients clinically N0 (cN0) at preoperative assessment, with unifocal primary tumor, $<4$ $\mathrm{cm}$, not previously excised nor subjected to neoadjuvant treatment. The cN0 patients unsuitable for these strict criteria currently undergo to radical lymphadenectomy, resulting unnecessary in the $70 \%$ of cases, due to a negative final histology. In our previous prospective trial (GroSNaPET study) we performed in this subgroup of patients the SNB followed by standard lymphadenectomy, demonstrating safety and accuracy of SLN (73 groins enrolled). In this report on the extended series including a total of 111 groins, we update the follow up data.

Methodology According to the Grosnapet study design, lymph node status was assessed by pre-operative PET/CT scan and cN0 patients were enrolled on the base of the following criteria: a) Tumor $>4 \mathrm{~cm}$, b) Multifocal tumors, c) previous complete excision; d) contralateral nodal involvement, e) previous RTCT treatment or $\mathrm{f}$ ) vulvar recurrence. Vulvar surgery was performed according to current recommendations, as appropriate. Sentinel lymph node was detected by radiotracer and blue dye to reach the maximum detection rate. Radical lymphadenectomy was always provided after SNB, according to the standard of care. Both PET/CT scan and SNB were compared to final pathology report. Patients were followed up quarterly, undergoing clinical visit, groin ultrasound and PET/CT scan.

Result(s)* During the study period, 72 patients were considered eligible for a total of 111 groins included. Median patient's age was 73yrs. Histopathology revealed 16 (14.4\%) groins with metastatic sentinel nodes (SLNs). Only one case had further involved non SLNs. Overall, 18/274 (6.5\%) SLN excised were positive at histology. Median metastasis diameter was 4.8 (1.5-12). One false negative SN was identified (NPV 99\%). PET/CT showed an NPV of 92\%. After a median follow-up of 38 months (range 1-97 months), 19 recurrences and 6 deaths were registered. The 3-year disease-free survival (DFS) was $72.6 \%$.

Conclusion* This is the largest series that strongly support the use of SNB in cN0 patients currently excluded. A careful preoperative study could safely select eligible patients. Further validation is advisable.

\section{VULVAR CANCER TREATMENT BETWEEN 2010 AND 2019: THE EXPERIENCE OF A SINGLE ROMANIAN CANCER CENTER}

${ }^{1} \mathrm{~V}$ Galatan*, ${ }^{1} \mathrm{~N}$ Todor, ${ }^{1,2} \mathrm{AT}$ Kirsch-Mangu, ${ }^{1,2} \mathrm{D}$ Pop, ${ }^{1} \mathrm{Cl}$ Trifan, ${ }^{1} \mathrm{C}$ Ordeanu, ${ }^{1} \mathrm{R}$ Bodea, ${ }^{1} \mathrm{C}$ Pop, ${ }^{1,2} \mathrm{O}$ Coza, ${ }^{1,2}$ VM Nagy, ${ }^{1,2}$ P Achimaş Cadariu, ${ }^{1,2} \mathrm{FL}$ Ignat, ${ }^{1,2} \mathrm{DT}$ Eniu, ${ }^{1,3} \mathrm{AP}$ Trăilă, ${ }^{1,2} \mathrm{G}$ Lazar, '\$ Hica, ${ }^{1,2} \mathrm{AC}$ Rancea, ${ }^{1,2} \mathrm{IC}$ Lisencu, ${ }^{1,2} \mathrm{IC} \mathrm{Vlad},{ }^{3} \mathrm{~A}$ Trăilă. ${ }^{1} T$ The Oncology Institute "Prof. Dr. Ion Chiricuță" Cluj-Napoca, Cluj-Napoca, Romania; ${ }^{2}$ University of Medicine and Farmacy "Iuliu Hatieganu" Cluj-Napoca, Romania, Cluj Napoca, Romania; ${ }^{3}$ General Hospital "Humanitas" Cluj-Napoca, Romania, Surgery, Cluj Napoca, Romania

\subsection{6/ijgc-2021-ESGO.634}

Introduction/Background* The purpose of this study was to evaluate 5-year overall survival (OS), disease free survival (DFS) and local control (LC) for patients diagnosed with primary vulvar cancer and treated at Institute of Oncology "Prof. Dr.Ion Chiricuta" Cluj-Napoca, Romania.

Methodology Between 2010 and 2019 a number of 306 patients with vulvar cancer were treated in our institution, from which we included in this retrospective study 233 patients with squamous cell vulvar cancer; based on FIGO staging: $19(8.2 \%)$ patients were stage 0,115 (49.4\%) stage I, $17(7.3 \%)$ stage II, $66(28.3 \%)$ stage III and $16(6.8 \%)$ 